\title{
Subscription Propagation in Highly-Available Publish/Subscribe Middleware
}

\author{
Yuanyuan Zhao, Daniel Sturman, and Sumeer Bhola \\ IBM T.J.Watson Research Center, \\ Hawthorne, NY 10532, USA \\ \{yuanyuan, sturman, sbhola\}@us.ibm.com
}

\begin{abstract}
Achieving availability and scalability while providing service guarantees such as in-order, gapless delivery is essential for deploying publish/subscribe messaging middleware in wide area networks. Scalability often requires a publish/subscribe system to propagate subscription information and perform content matching across the network. Existing subscription propagation algorithms do not support in-order, gapless delivery in a redundant overlay network.

This paper presents a novel approach that utilizes virtual time (VT) vectors to convey temporal consistency in propagating incremental and consolidated subscription information. The VT vectors provide a means of testing sufficiency of filtering information, by comparing a broker's VT vector with that of a message. When the test fails, indicating insufficient broker subscription information, safety may be preserved by 'flooding' the message to all neighbors on a routing tree. This approach does not require subscription state agreement across redundant paths and hence is highly available. We present a detailed evaluation of the approach.
\end{abstract}

Keywords: publish/subscribe middleware, subscription propagation, high availability

\section{Introduction}

Content-based publish/subscribe(pub/sub) messaging is a popular paradigm for building asynchronous distributed applications. A content-based pub/sub system consists of publishers that generate messages and subscribers that register interest in messages. The interest is usually expressed through content filters in the form of Boolean expressions. The system ensures timely delivery of published messages to all interested subscribers, and typically contains routing brokers for this purpose. Publishers and subscribers obtain service by connecting to brokers and are decoupled with each other.

In many cases, publishers and subscribers also want strong service guarantees, such as in-order, gapless delivery $[3,4]$ (referred to as reliable delivery in the rest of the paper). These services are usually provided by the broker network and made available to clients through standard messaging interfaces, like the Java Message Service (JMS) [1]. Reliable delivery guarantees that for every subscription $s$ and a published message stream, the system finds a starting message 
$m_{0}$ and from $m_{0}$, delivers all and only those messages matching $s$ in an order conforming to the original stream.

In addition to providing reliable delivery guarantees, commercial deployment over wide-area networks requires a pub/sub system to be scalable, highly available and utilize network bandwidth efficiently. In order to efficiently utilize network bandwidth, a pub/sub system usually propagates information about subscriptions across the broker network. A broker stores, for each of its neighboring part of the network, the information on what messages are needed by subscribers from the network. When a new message is published and routed, a broker filters out and does not send the message to parts of the network where no subscriber is interested. However, the amount of subscription information could get very large as it approaches the publishers. A scalable pub/sub systems should aggregate and only maintain a subset of subscription information for each routing direction of a broker as long as the subset of information is sufficient to match all messages that are needed by subscribers from that routing direction.

The combination of content-based routing and reliable delivery provides some unique challenges. Unlike in topic or group-based pub/sub systems, reliability cannot be based only on detecting gaps in publisher-assigned sequence numbers as each content subscriber may request a completely unique set of messages to be delivered. Reliable delivery protocols typically rely on brokers on the routing path to assist on detecting gaps. A routing broker with incorrect subscription information may decide not to forward on a message. Given that gaps cannot be detected by checking publisher-assigned sequence numbers, an end subscriber may never discover that a message was missed.

Numerous studies have shown that loss of connectivity is common in widearea networks, due to hardware and software failures and network misconfigurations. Hence, pub/sub systems should be built on networks with redundant links. This further complicates subscription propagation as alternative routes with different subscription information may filter out messages matching subscriptions that are unknown to a route.

In this paper, we present a subscription propagation algorithm that supports reliable delivery in redundant overlay networks. Using this algorithm, a pub/sub system is able to (1) propagate and aggregate subscriptions; (2) support reliable delivery; (3) choose freely (based on other criteria such as traffic load, system faults) among multiple redundant links for data message routing; and (4) not require heavy consensus protocols among brokers that serve as alternatives or backups of each other. Our approach deals with link failure, message losses and re-ordering and broker crash failures. Byzantine failures are outside the scope of this work.

We have implemented our algorithm in the Gryphon system. Gryphon is a content-based pub/sub system designed for high-volume, low latency, Internetscale distribution. Gryphon can be deployed using multiple geographically-distributed application-level routers (called brokers), with tens of thousands of clients and with tens of thousands of messages being delivered across the sys- 
tem each second. Our algorithm, however, applies to a wide range of messaging systems.

Previous pub/sub systems have mainly focused on efficiently utilizing network bandwidth and on scalability issues concerning propagating and aggregating subscriptions. However, few of these systems efficiently exploit the network redundancy and recover from failures in a timely fashion. Indeed, existing pub/sub systems are usually unable to utilize the redundant links or do not support reliable delivery. Simply extending existing work that utilizes only a single path requires consensus between multiple brokers on alternative paths and will incur a lot of overhead.

The remainder of this paper is organized as follows: Section 2 presents a redundant overlay network model. Section 3 describes the basic algorithm, and then discusses a number of variants. Section 4 discusses liveness and failure recovery, and Section 5 describes our implementation and experimental results. Section 6 is an overview of related work and we conclude in Section 7.

\section{Context: Redundant Overlay Network}

In this section, we describe a simplified topology model of a redundant overlay network of messaging brokers. We assume a redundant routing tree where each tree node may contain more than one broker/server(Figure 1). We further restrict the edge nodes (e.g. $N_{11}, N_{12}, N_{13}$, and $N_{31}$ ) to contain only one broker. We assume there are bi-directional links between any two brokers in neighboring nodes. Furthermore, we assume brokers residing in the same tree nodes are fully connected when there is no failure. The algorithm, however, does not depend on the full connectivity.

As we describe the protocol from the standpoint of a single publisher connecting broker, we arbitrarily designate an edge node as the root of the tree and other edge nodes as the leaves. Publishers connect at the root broker (also called publisher connecting broker) and subscribers at leaf brokers (subscriber connecting brokers). The full algorithm with publishers/subscribers connecting at any edge node is a repetition of this simplified description with repetitive information avoided. As a convenience, we refer to where the root node resides as upstream, and leaf nodes as downstream.

The singleton broker in an edge node can be replicated but requires strong consistency to ensure client connection and failover from one replica to another does not cause message loss or duplication.

Figure 1 shows a redundant routing tree that contains: 2 non-edge nodes $N_{21}$ and $N_{22}$ each with two brokers; 1 root node $N_{31}$ with broker $P B_{1}$ for publisher connections; and 3 leaf nodes $N_{11}, N_{12}$ and $N_{13}$ each with one broker $\left(S B_{1}, S B_{2}\right.$ and $S B_{3}$, respectively) for subscriber connections.

This model may seem a bit restrictive at first glance. However, the brokers referred here are logical brokers. A broker process may represent multiple logical brokers and hence participate in more than one node and have virtual links between its multiple presence in neighboring tree nodes. Furthermore, publishers 


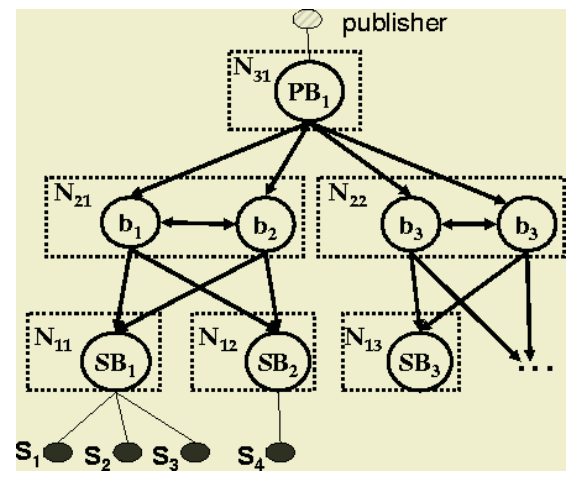

Fig. 1. A Redundant Routing Tree.

and subscribers can connect to any physical brokers. The physical broker in this case implements a logical leaf broker. Thus we abstract the topology to have publishers and subscribers connecting only at the edge nodes. How to map physical brokers to logical brokers, and what brokers reside in which tree nodes is an important part of the Gryphon technology. We do not describe it here.

\section{Algorithms}

\subsection{Solution Intuition}

To properly introduce the intuition behind our algorithm, we revisit in detail the challenges facing subscription propagation. We use the example network shown in Figure 1 to illustrate.

One challenge is to decide the delivery starting point of a subscription, that is, to find a position in a published message stream from which the system can deliver messages in order and without gaps. This problem is complicated because the new subscriptions may partially overlap with existing subscriptions. Suppose in the network in Figure 1, subscriber/subscription $s_{1}$ of stock $=$ nyse $: i b m$ has been propagated to all brokers in the tree nodes on the path from $N_{11}$ to $N_{31}$, that is, broker $S B_{1}, b_{1}, b_{2}$ and $P B_{1}$. When subscriber $s_{2}$ submits subscription stock $=$ nyse $: *$ (which matches all messages matching $s_{1}$ and more), broker $S B_{1}$ propagates this information toward $P B_{1}$. However, when message $m_{1}$ with content (nyse : ibm,92) arrives, broker $S B_{1}$ does not know whether it should deliver it to subscription $s_{2}$ as well. It might be an error to deliver $m_{1}$ because $m_{1}$ arrives only as a result of matching subscription $s_{1}$. If this is the case, there is no guarantee that a later message $m_{2}$ of (nyse $\left.: t, 19\right)$ will be routed by the system and thus result in a gap in the messages delivered to subscription $s_{2}$.

Another challenge arises when messages that are part of the same ordered stream travel along different paths. This might be a result of a load sharing decision or a failed link. Furthermore, the message delivery path could be different from the path subscription has been propagated. For example, subscription 
$s_{2}$ might have been propagated from $S B_{1}$ to $b_{1}$ and $P B_{1}$ but not yet to $b_{2}$. If message $m_{1}$ is routed through broker $b_{1}$ and $m_{2}$ through $b_{2}, b_{2}$ would not send $m_{2}$ to $S B_{1}$ without knowing subscription $s_{2}$. Delivery for $s_{2}$ might have already started and $S B_{1}$ would not detect the gap created by missing $m_{2}$. This kind of gap can not be detected by the reliable delivery protocol because it relies on correct subscription information.

Our algorithm solves these problems by making two types of information explicit, i.e., the set $S_{m}$ of subscriptions a message should be matched against and the set $S_{b}$ of subscriptions a broker has information of and uses for message matching. Using this information, delivery starting point of a subscription can be detected by choosing the first message whose $S_{m}$ set contains the subscription. In addition, the system guarantee that for every message published in the same stream after the first message, its $S_{m}$ set (eventually) includes the subscription until it is unsubscribed. How and when the $S_{m}$ set of a message is assigned and changed is a dimension in which an algorithm can vary.

To prevent a broker (e.g. $\left.b_{2}\right)$ to filter out a message by mistake, the broker compares its $S_{b}$ set with the $S_{m}$ set of the message. The broker contains information of the subtree where a subscription in $S_{b}$ set is connected and thus can project/partition the $S_{b}$ set onto each subtree rooted at the tree node where the broker resides. The broker can also project the $S_{m}$ set onto each subtree but only partially because the $S_{m}$ set may contain subscriptions that is not in the broker's $S_{b}$ set. The comparison of $S_{m}$ and $S_{b}$ is thus performed for each subtree rooted at a child node (e.g. $N_{11}$ ). It is possible that in subtree $N_{11}$ there is a subscription that is contained in $S_{m}$ but not in $S_{b}$, and the subscription matches the message. However, since the broker does not have information about the subscription, its filtering result will probaby indicate that the message does not need to be sent to subtree $N_{11}$. Our algorithm takes the comparison result of $S_{m}$ and $S_{b}$ as an indicator that whether the broker matching/filtering result should be used. If $S_{b}$ is the same or a superset of $S_{m}$ for a subtree, the broker contains sufficient information to perform matching and can decide whether or not to send the message to a subtree based on the existence of matching subscriptions. Otherwise, as a conservative measure, the message should be sent to the subtree regardless of the matching result. We call this comparison of $S_{b}$ and $S_{m}$ sets the sufficiency test.

With this type of algorithm, there is no need to maintain consistent states of subscription information between redundant path brokers. As a result, the solution is light-weight and highly available.

\subsection{Solution Overview}

Obviously, concise representation of $S_{m}$ and $S_{b}$ sets and efficient computation of the sufficiency tests are crucial for system performance. In our approach, each leaf broker maintains a virtual time clock. We assign each subscription a virtual start time (vst) at its subscribing time and a virtual end time (vet) at its unsubscribing time using the current value $(V T)$ of the virtual clock at the leaf broker to which the subscription connects. We call a subscription that has not 
unsubscribed active. Such a subscription has a vet that is greater than the VT of the leaf broker to which the subscriber is connected. The virtual clock advances whenever necessary and more than one subscription/unsubscription can occur in a single virtual time tick.

Thus, we can represent the set $S_{m}$ of a message as a vector $V_{m}$, with one element per leaf broker. Assume a $V_{m}$ vector $\left(S B_{1}: v_{1}, \cdots, S B_{n}: v_{n}\right)$, the set $S_{m}$ of the message is "any active subscription at broker $S B_{1}$ with $v s t$ no later than $v_{1}$ and vet later than the current virtual time $V T_{1}, \cdots$, plus any active subscription at broker $S B_{n}$ with $v$ st no later than $v_{n}$ and vet later than the current virtual time $V T_{n} "$.

Similarly, we represent the set $S_{b}$ of a broker as a vector $V_{b}$, with one element per leaf broker. Assume a $V_{b}$ vector $\left(S B_{1}: v_{1}^{\prime}, \cdots, S B_{n}: v_{n}^{\prime}\right)$, the set $S_{b}$ of the broker is "subscriptions at broker $S B_{1}$ with vst no later than $v_{1}^{\prime}$ and vet later than $v_{1}^{\prime}, \cdots$, plus subscriptions at broker $S B_{n}$ with $v$ st no later than $v_{n}^{\prime}$ and vet greater than $v_{n}^{\prime} "$.

Because virtual time is generated from a leaf broker's virtual clock, $v_{i}^{\prime} \leq V T_{i}$ $(i=1 . . n)$. If $v_{i}^{\prime} \geq v_{i}(i=1 . . n), S_{b} \supseteq S_{m}$. Thus, the sufficiency test can be efficiently implemented as comparison of $V_{b}$ and $V_{m}$ on the relevant elements. That is, for a downstream where certain subscriber-connecting/leaf brokers exist, the sufficiency test succeeds when $V_{b}$ is element-wise no less than $V_{m}$ for those brokers. In Figure 1, when broker $b_{1}$ or $b_{2}$ routes a message to tree node $N_{11}$, the sufficiency test succeeds when its $V_{b}$ is no less than $V_{m}$ for the element of $S B_{1}$. When broker $P B_{1}$ in node $N_{31}$ routes a message toward $N_{21}$, the sufficiency test succeeds when its $V_{b}$ is no less than $V_{m}$ for the elements of both $S B_{1}$ and $S B_{2}$.

In the remainder of this section, we describe in detail how subscriptions are assigned virtual start and end times, how subscription information is propagated, how messages are routed and how the system decides a message delivery starting point for a subscription. We discuss in Section 5.1 the optimization techniques to avoid sending full $V_{m}$ vectors in messages and to cache sufficiency test results.

\subsection{Assigning Subscription Virtual Start/End Times}

Clients submit subscriptions to a pub/sub system through their connecting brokers. A broker assigns a virtual start time to a subscription during its subscribing process. As mentioned in Section 2, these brokers are in the leaf nodes of the redundant routing tree. A leaf broker usually aggregates subscriptions and only propagates to upstream the aggregated results.

A client subscription/unsubscription may or may not change the aggregated subscriptions of its connecting broker, depending on existing subscriptions. Whenever a leaf broker's subscriptions change and the broker decides to propagate the change to upstream, the broker advances its virtual time clock by 1 . The virtual clock is maintained in an integer counter and is also used to assign virtual start/end times to subscriptions.

The leaf broker ensures the monotonicity of its virtual clock, despite crash recovery. There are many possible techniques to achieve this, such as using a monotonic system clock, or persisting an upper bound on the highest clock value. 


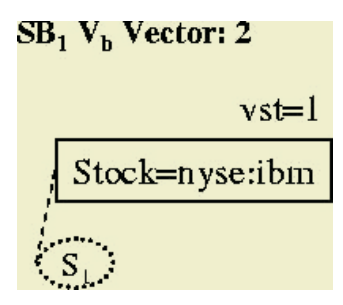

(a) Sub DAG w/ $s_{1} \& s_{4}$

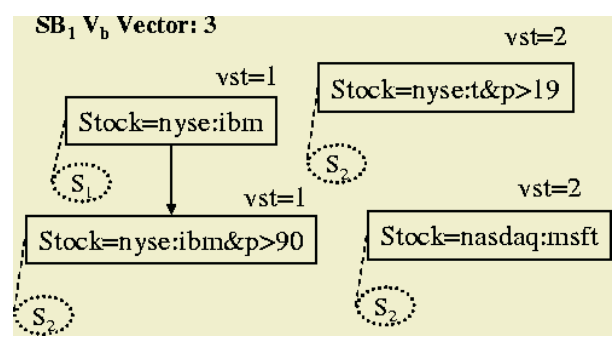

(b) Sub DAG w/ $s_{1} \& s_{4} \& s_{2}$

Fig. 2. Broker $S B_{1}$ Subscription DAGs.

We assume that the clock time never overflows, which is reasonable for a value that is 64 bits.

We assume that a client subscription is in the form of a set of Boolean conjunctions. Any other form of Boolean expression can be transformed into disjunctive normal form (DNF). We define the following "covering" relationship of conjunctions similar to that of [5].

Definition 1. Conjunction $c_{1}$ covers $c_{2}$, denoted as $c_{1} \succeq_{c} c_{2}$, if and only if all messages matching $c_{2}$ also match $c_{1}$. That is

$$
c_{1} \succeq_{c} c_{2} \Leftrightarrow\left\{M\left(c_{1}\right) \supseteq M\left(c_{2}\right)\right\}
$$

where $M(c)$ is the set of all messages matching conjunction $c$.

Conversely, $c_{2}$ is said to be "covered by" $c_{1}$.

Subscribing Process. To assign a virtual start time to a new subscription, we analyze the covering relationship of its conjunctions with the existing subscription conjunctions. We use a directed acyclic graph (DAG) by modeling conjunctions as nodes and drawing a directed edge from a covering conjunction to a covered conjunction. As the covering relationship is transitive, we omit the transitive edges. Initially, broker conjunction DAGs are empty. Examples of conjunction DAGs are shown in Figure 2 and 3. Each conjunction is represented as a rectangle, with a virtual start time and an oval representing the list (called subscriber list) of subscribers or downstream routing tree nodes whose subscriptions contain the conjunction.

For each conjunction $c$ of the new subscription, the broker finds existing conjunctions that cover $c$ and set c.vst to the minimum of these conjunctions. If no covering conjunction exists, c.vst is set to the broker's current virtual time.

To ensure reliable delivery, message delivery for a new subscription cannot start until matching messages for all its conjunctions start to arrive, hence vst of the new subscription is set to the maximum of its conjunctions'. The new conjunction nodes are added into the conjunction DAG.

Example 1. Suppose clients in Figure 1 submit the following subscriptions:

1. $s_{1}:$ stock $=$ nyse $: i b m\left(\right.$ submitted at $\left.S B_{1}\right)$

2. $s_{4}:$ stock $=$ nyse $: *\left(\right.$ submitted at $\left.S B_{2}\right)$

3. $s_{2}:$ stock $=$ nyse $: i b m \& p>90$ or stock $=$ nyse $: t \& p>19$ or stock $=$ nasdaq : msft (submitted at $S B_{1}$ ) 


\section{$b_{1} V_{b}$ Vector: $\left(S B_{1}: 1, S B_{2}: 1\right)$}

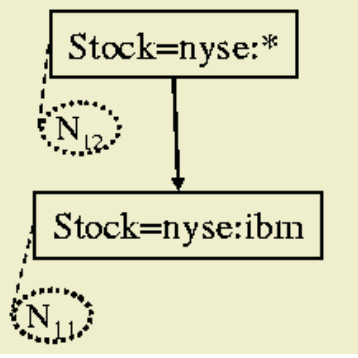

(a) Sub DAGw/ $s_{1} \& s_{4}$

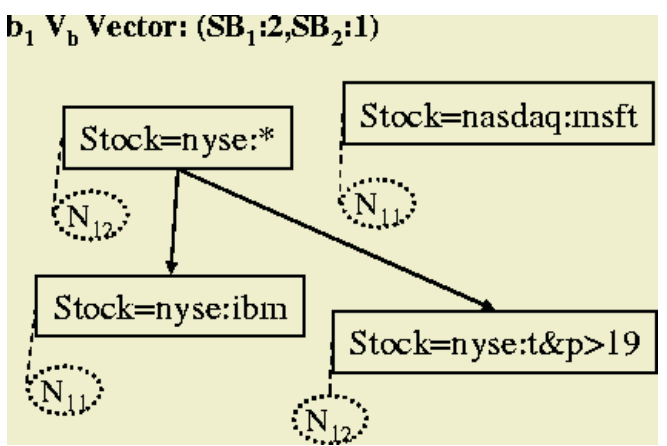

(b) Sub DAG w/ $s_{1} \& s_{4} \& s_{2}$

Fig. 3. Broker $b_{1}$ Subscription DAGs.

The conjunction DAGs and $V_{b}$ vectors of broker $S B_{1}$ and $b_{1}$ after subscription $s_{1}$ and $s_{4}$ are shown in Figure 2(a) and 3(a). When $s_{2}$ is subscribed, its first conjunction is covered by the only node in the conjunction DAG (Figure 2(a)) and thus has a vst of 1 . The other two conjunctions are not covered and thus have vst of 2 . Hence $s_{2} . v s t=2 . S B_{1}$ increments its $V T$ to 3 and changes the conjunction DAG to Figure 2(b).

It is interesting to observe that the nodes in the conjunction DAG are assigned non-increasing vst's as one travels from a root node to the covered nodes. Thus computing vst of a new conjunction only takes into consideration the immediate covering nodes. As a reminder, transitive arcs are omitted from the conjunction DAG.

Unsubscribing Process. During client's unsubscription of its conjunctions, the client is removed from the subscriber lists of the nodes in the conjunction DAG. Conjunctions with empty lists are removed from the DAG. The subscription is assigned a virtual end time using the broker's current virtual time. Note that since a subscription is removed, there is no need to store information for it. The vet is not really assigned and is never maintained in the system. We only use it for convenience in describing the subscription sets $S_{m}$ and $S_{b}$.

At the end of the client subscribing/unsubscribing process, if any roots are added or removed, the leaf node broker advances its virtual clock by one. In addition, an incremental update is generated and propagated to upstream brokers in the redundant routing tree.

We should note that although we have discussed the subscribing and unsubscribing process as if there is only one request at a time, the algorithm actually processes requests in batches. The broker only advances its virtual clock at the end of the batch process if the set of root nodes is changed.

\subsection{Propagating Subscription Changes}

As described previously, if client subscription/unsubscription results in changes in the set of root nodes of a conjunction DAG, the leaf broker generates and 
propagates incremental changes. An incremental change contains the name of the originating leaf broker $S B_{1}$, the virtual time $v_{1}$ of $S B_{1}$ when the change occurred, a list of additive/subtractive conjunctions $\left\{+c_{1},+c_{2}, \cdots,-c_{i},-c_{i+1}, \cdots\right\}$, the subscribing tree node, and a constraint vector on receiving broker's $V_{b}$ vector. $c_{1}$ and $c_{2}$ are the new root DAG nodes. $c_{i}$ and $c_{i+1}$ are the old roots that were just removed from the DAG. The constraint vector is initially $\left(S B_{1}, v_{1}-1\right)$. That is, we require a broker have information of all subscriptions connected at leaf broker $S B_{1}$ with $v s t<v_{1}$.

Example 2. Continuing with Example 1, the incremental change $S B_{1}$ generated as a result of $s_{2}$ subscription is:

$S B_{1}, 2,\{+($ stock $=$ nyse $: t \& p>19),+($ stock $=$ nasdaq $:$ msft $)\}, N_{11}$, cons $=$ $\left(S B_{1}: 1\right)$.

The leaf broker sends the incremental change to a broker in its parent node, e.g., broker $b_{1}$ in $N_{21}$ in Figure 1 . As brokers in the same tree node are usually fully connected, $b_{1}$ forwards the incremental change to other brokers $\left(b_{2}\right)$ in $N_{21}$. How this incremental change is propagated to all brokers in the parent node is not fixed and a specific algorithm may adapt if the topology assumptions are different.

Upon receiving an incremental change, a broker checks whether its $V_{b}$ vector satisfies the constraint. If so, it updates its $V_{b}$ vector and applies the additive/subtractive conjunctions by adding/removing the tree node (e.g., $N_{11}$ ) to/from the subscriber lists of conjunction nodes and inserting new conjunctions or removing conjunctions with empty subscriber lists from the DAG. A non-leaf node broker's conjunction DAG (e.g., Figure 3(a) and (b)) is similar to that of a leaf node broker's, except that vst's are not recorded for conjunctions. Same as in a leaf broker, the additive/subtractive conjunctions in the new incremental change are computed as a result of the root node changes in the DAG. If the incremental change is a pure additive change and no aggregation happened in the current broker, the constraint vector of the new change is unchanged. Otherwise, the constraint vector is set to the old $V_{b}$ vector of the broker. The subscribing tree node of the new incremental change is set to the tree node where the current broker resides. The original receiving broker of the incremental change then forwards the new incremental change to a broker in its parent tree node.

If the constraint is not satisfied, the broker cannot apply the incremental change. Furthermore, if some elements of the broker's $V_{b}$ vector are smaller than that of the constraint vector, the broker detects its subscription information is lagging behind, and initiates liveness mechanism to get up-to-date (described in Section 4).

Example 3. In our example, broker $b_{1}$ satisfies the constraint vector of the incremental change. As conjunction stock $=$ nyse $: t \& p>19$ is covered by an existing conjunction stock $=$ nyse $: *$, it is aggregated away. Hence the new incremental change is:

$S B_{1}, 2,\{+($ stock $=$ nasdaq $:$ msft $)\}, N_{21}$, cons $=\left(S B_{1}: 1, S B_{2}: 1\right)$

$b_{1}$ 's $V_{b}$ vector is set to $\left(S B_{1}: 2, S B_{2}: 1\right)$. 
In our examples below, we use a vector such as $(1,1,1)$ to represent $V_{m}$ or $V_{b}$ vectors without mentioning the leaf broker names $S B_{1}, S B_{2}$ and $S B_{3}$.

Similar things happen at the root broker in the routing tree. Continuing with our example, broker $P B_{1}$ updates its $V_{b}$ vector from $(1,1,1)$ to $(2,1,1)$.

\subsection{Data Message Routing}

Data messages are published through the root broker (e.g., $P B_{1}$ ) of the routing tree. Before sending a newly published message, $P B_{1}$ assigns to it a $V_{m}$ vector. How this $V_{m}$ vector is assigned is a dimension in which the algorithm could vary and is further discussed in Section 3.7. For now, we assume $P B_{1}$ keeps a persistent record of the highest $V_{m}$ vector it has ever assigned and ensures non-decreasing $V_{m}$ 's for new messages. When $V_{b} \geq$ highest $V_{m}, V_{b}$ is used.

For an incoming message, a broker performs matching to decide which downstream routing tree nodes it should send the message to. Many efficient matching algorithms exist such as [2] [8]. Our algorithm works with any of them.

Furthermore, the broker compares its $V_{b}$ vector with the $V_{m}$ vector of the message for each child node. It does so by slicing both vectors with only the elements for the leaf brokers in the subtree rooted at the child node. If the $V_{b}$ vector is no less than the $V_{m}$ for the slicing, the broker sends the message if and only if the matching results show a match for the downstream. Otherwise, the broker conservatively sends the message down, regardless of the matching result. This is the sufficiency test we have mentioned in Section 3.1.

As we can see, it is possible for the broker to send down messages that do not match any subscription. This only happens at non-leaf brokers. In the leaf broker, as it always has the latest subscription information, its $V_{b}$ vector (containing only one element for itself) always satisfies the sufficiency test and only the matching messages will be delivered to subscribers.

Example 4. Suppose $P B_{1}$ assigns $V_{m}$ vector $(1,1,1)$ to message $m_{1}$ (nyse $: i b m$, $95)$ and $m_{2}$ (nyse : $\left.t, 20\right) \cdot m_{1}$ is sent to $b_{1}$ and $m_{2}$ to $b_{2}$. Suppose $V_{b}$ vectors of $b_{1}$ and $b_{2}$ are $(2,1,0)$ and $(1,1,0)$. Both messages will be sent to $S B_{1}$ as the sufficiency tests are satisfied for $S B_{1}$ and there is a match from node $N_{11}$.

Suppose now $P B_{1}$ processes an incremental change and advances its $V_{b}$ vector to $(2,1,1)$. On the other hand, $b_{2}$ does not receive the incremental change and thus its $V_{b}$ vector stays at $(1,1,0) . P B_{1}$ assigns $V_{m}$ vector $(2,1,1)$ to $m_{3}$ (nyse: $i b m, 98)$ and $m_{4}$ (nyse $\left.: t, 22\right)$ and sends $m_{3}$ to $b_{1}$ and $m_{4}$ to $b_{2}$. Both broker $b_{1}$ and $b_{2}$ send the messages to $S B_{1}$ because $b_{1}$ has a match and $b_{2}$, even though without a match for $N_{11}$, detects its sufficiency test fails.

Justification of Correctness. The sufficiency test is satisfied when a broker's $V_{b}$ vector is equal to or greater than $V_{m}$ with regard to the relevant leaf brokers. When it is greater, the broker can have wider (matching more messages) conjunctions as new subscriptions may have happened. It can also have narrower conjunctions as unsubscriptions may have happened. When the conjunctions are wider, the broker obviously passes all messages matching the subscriptions required by the $V_{m}$ vectors plus more that match the new subscriptions. In the 
narrower case, a broker drops messages matching only unsubscribed subscriptions at a leaf broker $s b$. Those subscriptions have vet $\leq V_{b}(s b) \leq V T_{s b}$ and hence are not in the $S_{m}$ set.

\subsection{Detecting Subscription Delivery Starting Point}

For a new subscription, its connecting leaf broker must decide a safe point from which the system can deliver a gapless, in-order stream of published messages. The actual protocol for reliable delivery is a complicated scheme and has been discussed in our work in [3] [4]. We do not deal with that problem here, rather we deal with how subscription propagation will not produce subscription information that is wrong for reliable delivery. Our solution can work with any reliable delivery protocol.

Detecting delivery starting point is accomplished by comparing a message's $V_{m}$ vector element with the $v s t$ of the subscription $s$. As soon as the leaf broker $s b$ sees a message with $V_{m}(s b) \geq$ s.vst, it starts to deliver matching messages for $s$.

Example 5. Broker $S B_{1}$ receives $m_{1}$. Even if $m_{1}$ matches subscription $s_{2}$, this is not a delivery starting point for $s_{2}$ as $m_{1}$ 's $V_{m}\left(S B_{1}\right)==1$ and is less than $s_{2} . v s t==2$. This is correct because there is no guarantee a later message (nyse : $t, 20)$ will not be filtered out if it is routed through broker $b_{2}$. Broker $S B_{1}$, instead, starts delivery for $s_{2}$ from message $m_{3}$.

\subsection{Algorithm Variants}

We have extended our basic algorithm with two variants. These extensions continue to support reliable delivery. Due to space restriction, we only provide brief description of these extensions:

- Non-monotonic assignment of $V_{m}$ vectors to messages in a published stream;

- Non-fixed $V_{m}$ vector for a message $m$.

Our basic algorithm stores persistently the highest $V_{m}$ assigned and assigns monotonically non-decreasing $V_{m}$ vectors to messages at the publisher connecting brokers. Our extension does not require persistent storage and allows nonmonotonic $V_{m}$ vector assignments to messages. This could happen due to a publisher connecting broker crash/recovery.

In the basic algorithm, a message's $V_{m}$ vector is fixed once assigned. In some cases, the aggregation of subscriptions may result in incremental updates with empty lists of additive/subtractive conjunctions. The basic algorithm requires such empty updates to propagate to the root broker as a means of conveying the latest $V_{m}$ the broker could use. Our extension does not require propagation of this kind of update, rather we record the fact that a $V_{m}$ vector could be automatically changed to $V_{m}^{\prime}$ due to the empty incremental update. This is recorded at the last broker where such an empty update occurred. 
In both extensions, not only are published messages assigned $V_{m}$ vectors, so are the silence periods between them. In addition, the leaf brokers hosting subscribers perform monotonicity checking on message/silence $V_{m}$ vectors and initiate negative-acknowledgments if monotonicity is violated.

\section{Liveness and Failure Handling}

Our solution intrinsically supports pub/sub message delivery with high availability and light failover. The solution itself has to deal with failures as incremental subscription updates can be lost and arrive out-of-order. This section describes the failure handling with regard to the subscription propagation protocol. This is an important part of our work, however, due to space restriction, we only describe it briefly.

Broker Crash Recovery. Upon recovery from a crash, a leaf broker sets its virtual clock time $V T$ to be greater than all previous values it has assigned. The leaf broker initializes its conjunction DAG to contain conjunctions for the durable subscriptions ([1][4]) it maintains. All conjunctions are then propagated by sending a full state update and the current $V T$. The broker then advances its $V T$ by one.

Non-leaf brokers recover from a crash by initializing an empty conjunction DAG and resetting its $V_{b}$ vector to all 0's.

Leaf Broker Driven Liveness. As the sources of subscription changes and virtual times, leaf brokers ensure all publisher connecting brokers receive up-to-date subscription information and assign latest $V_{m}$ vectors to messages. For subscription/unsubscription received during virtual time $v t$ and propagated, a leaf broker $S B$ maintains an expected starting time from which data messages should have $V_{m}$ vector with an element $v t^{\prime}$ for $S B$ such that $v t^{\prime} \geq v t$. This expected time can be dynamically adjusted through similar techniques that estimates TCP round trip times. Messages received after the elapsed time with $v t^{\prime}<v t$ trigger a full subscription state update with $S B$ 's latest propagated $V T$. Alternatively, $S B$ can repeat the incremental updates sent from $v t^{\prime}$ to $v t$. This requires a cache for the latest incremental updates at $S B$.

Non-leaf Broker Driven Liveness. A non-leaf broker (including publisher connecting brokers) $b$ detects that its subscription information is lagging behind in two ways: (1) receives a data message with $V_{m}$ vector that is greater than $V_{b}$ vector for the elements of downstream leaf brokers; (2) receives incremental updates with constraint vectors $\not \leq V_{b}$. Broker $b$ initiates a negative acknowledgment message toward the leaf broker $S B$ for whom $b$ 's $V_{b}$ vector is lagging behind. Such negative acknowledgment may be satisfied by $S B$ or a broker on the route from $b$ to $S B$ with the required subscription information. 


\section{Implementation and Experimental Results}

\subsection{Implementation}

Our implementation performs monotonic and fixed $V_{m}$ vector assignment at the publisher connecting brokers. These brokers persist the highest $V_{m}$ vector assigned. The liveness and failure handling utilizes full-update messages.

Subscription aggregation is based on covering relations (Section 3). Our current covering relation is restricted to identical conjunctions. Since the covering test is a black-box component in our implementation, a sophisticated covering relation can be plugged in to replace this simple one.

The number of leaf brokers affects system performance and scalability, since it directly impacts the length of $V_{m}$ and $V_{b}$ vectors and computations involving them. In our implementation, we utilize the property that links are usually FIFO. This is true of links implemented as TCP connections such as in Gryphon.

Instead of full $V_{m}$ vectors, we use fixed-length vector digests. When a new $V_{m}$ is first assigned to a message, the publisher connecting broker assigns a digest to $V_{m}$ by taking a snapshot of a monotonically increasing value. This can be implemented in many ways, such as using the system clock. As publisher connecting broker assigns monotonically non-decreasing $V_{m}$ vectors and digests are monotonic, the system satisfies the following monotonicity $-d<d^{\prime}$ if $V_{m}<$ $V_{m}^{\prime}$ with regard to the same assigning broker. A broker does not persist the digests, therefore it can assign two different digests to one $V_{m}$ at different times. Thus $d<d^{\prime} \Rightarrow V_{m} \leq V_{m}^{\prime}$.

In this scheme, the first message with a new $V_{m}$ vector going down each link carries the original $V_{m}$ and its digest. Later messages only need to carry the digest until the $V_{m}$ changes or the link fails and recovers.

In addition, each broker maintains a cache of the sufficiency test results. This cache is indexed by the digest and the assigning broker. It only maintains an entry for the highest digest it has seen from a publisher connecting broker. From the digest monotonicity, any message originated from the same broker with a digest no greater than the cache entry can reuse the cache result. Otherwise, the broker conservatively computes false as the sufficiency test result for the message. The cache entry is updated when the broker advances its $V_{b}$ vector or when it receives a message with higher $V_{m}$ vector and digest. We omit the details of a negative-acknowledgement scheme that is used to retrieve the original $V_{m}$ vector for a digest if the message carrying the mapping was lost.

\subsection{Experiment Results}

The testbed for our experiments is a set of RS6000 F80 machines with six $500 \mathrm{MHz}$ processors and $1 \mathrm{G}$ RAM. Each machine has dual network interfaces and is connected through a 100Mbps Ethernet network and a gigabit switch to other machines.

We focus primarily on metrics that are impacted by the specifics of our solution. Metrics such as routing table (conjunction DAG) size and subscription incremental update message size are common to subscription propagation algorithms and have been investigated in previous work such as $[12,5]$. 

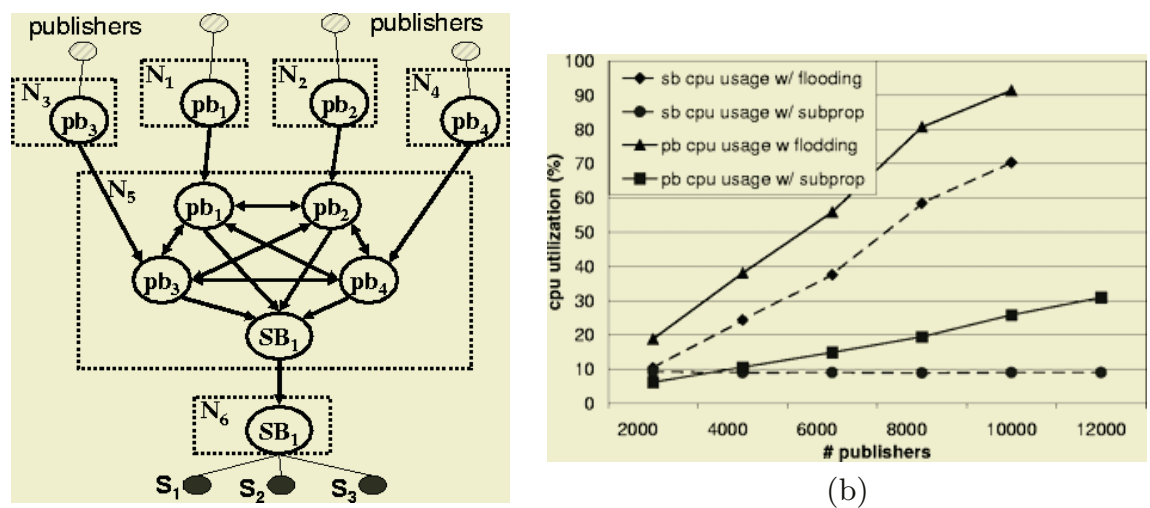

(b)

(a)

Fig. 4. System Load(CPU) Comparisons.

System Load Comparison in Selective Subscription Tests. This test compares the system overhead of using subscription propagation with that of using flooding when client subscriptions are selective. The workload is motivated by sensor networks where there are many publishers collecting and publishing various kinds of data and relatively few subscribers that selectively subscribe to data of interest.

The test is set up as Figure 4 (a) with 4 publisher connecting brokers $p b_{1-4}$ and 1 subscriber connecting broker $s b$, each in its own tree node. These brokers also reside in an intermediate tree node $N_{5}$ and thus each implements two virtual brokers. Four redundant routing trees can be defined by taking each of $p b_{1-4}$ as root node.

We fix the number of subscriptions at 2000 and vary the number of publishers from 2000 to 12000 . The message rate per publisher is fixed at 2 messages/second. Each subscription is distinct and to the messages published by 1 publisher. Hence, the total receiving message rate of subscribers is 4000 messages/second throughout the test. The publishers are evenly distributed among $p b_{1-4}$. Figure $4(\mathrm{~b})$ shows the CPU utilization of $s b$ and $p b_{1-4}$ (averaged) in both schemes.

With 2000 publishers, all published messages are subscribed. This case is not favorable to subscription propagation. However, CPU utilization difference at $s b$ in flooding and subscription propagation schemes are negligible due to the use of efficient matching algorithm [2]. In addition, when the number of publishers increases, $s b$ CPU utilization stays constant in the subscription propagation scheme but increases linearly in flooding scheme even though the number of useful messages does not change.

In both schemes, CPU utilization at $p b_{1-4}$ increases linearly with the number of publishers. However, the flooding scheme shows a much steeper slope because each of $p b_{1-4}$ not only accepts messages from publishers but also sends these messages to other $p b$ 's and receives all messages published through other $p b$ 's. 
As a result, CPU utilization at $p b_{1-4}$ reaches $>90 \%$ with only 10000 publishers in the flooding scheme compared to $31 \%$ with 12000 publishers with subscription propagation.

Latency Measurements. This test examines two latency metrics. Message delivery start latency ( $D S L a t)$ measures the time elapsed from a subscription is submitted to the first message is delivered to it. Message delivery latency (DLat) measures the time it takes the system to deliver a message to an existing subscription. We use a linear topology consisting of broker $p b$ for publishers and $s b$ for subscribers each in its own routing tree node. $p b$ and $s b$ are connected through $n$ hops of intermediate tree nodes, each with one broker.

DLat is measured by a latency sampler $(L S)$ that publishes messages through $p b$ and subscribes to its own messages through $s b$.

DSLat for a new subscription is measured at the subscriber by taking the difference between time of subscription and time of first message delivery. We further distinguish DSLat for subscriptions that are covered locally in $s b$ and subscriptions that are not covered hence must propagate to $p b$. We call the first DSLat local and the latter global. Global DSLat is the sum of the following times: (1) time taken to send subscription to $s b ;(2)$ processing time at $s b ;(3)$ processing time at other brokers; (4) network delays(bi-directional) at each hop; (5) expected interval till next message published that matches the subscription. If messages that match the subscription are published at a steady rate every $t$ milliseconds (ms), this time is $t / 2$ on average; (6) time taken to send the message from $s b$ to the subscriber. Similarly, local DSLat is the sum of (1),(2),(5),(6).

We make time (5) negligible by using a high publishing rate (200 messages/second/topic) but on few (16) topics. The aggregated publishing rate is 3200 messages/second. In addition, since we are mainly interested in measuring processing overhead (2) and (3), we do not inject additional latency on the links. Since we are running in a LAN environment, (1), (4) and (6) are small. We impose load on $s b$ by connecting 60 steady subscribers to 8 topics. Local DSLat is measured by subscribers that dynamically subscribe and unsubscribe to the same 8 topics as the steady subscribers and global DSLat is measured by subscribers that dynamically subscribe to the remaining 8 topics. We take the median of all measurements. Figure 5 shows DLat, local and global DSLat when there are 1,4 and 7 hops from $p b$ to $s b$.

Message delivery latency DLat increases linearly from 3 to 6 to $9 \mathrm{~ms}$. Local DSLat-delivery start latency for covered subscriptions - stays roughly constant at $17 \mathrm{~ms}$. Global DSLat increases linearly from $26.5 \mathrm{~ms}$ to $36 \mathrm{~ms}$ to $45 \mathrm{~ms}$. The differences of global and local DSLat, which shows the network latency and broker (other than $s b$ ) processing overhead also increases from $9.5 \mathrm{~ms}$ to $20 \mathrm{~ms}$ to $28 \mathrm{~ms}$, which shows that subscription processing overhead is small.

Scalability Measurements. This test examines the system scalability with regard to the $V_{m}$ and $V_{b}$ vector sizes, i.e., number of subscriber connecting brokers. The test is set up with a broker $p b$ for publishers and a broker $i b$ connecting 


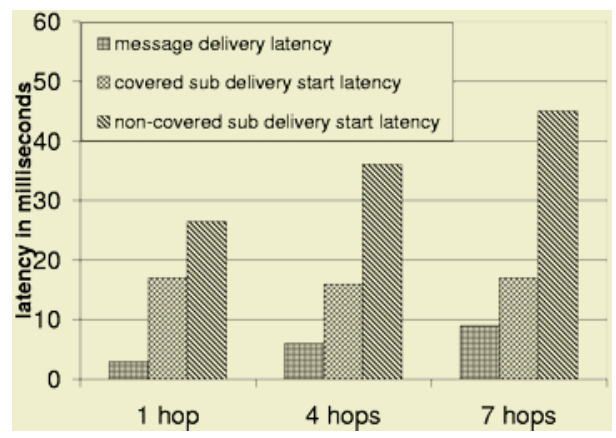

Fig. 5. Subscription Delivery Start Latency \& Message Delivery Latency.

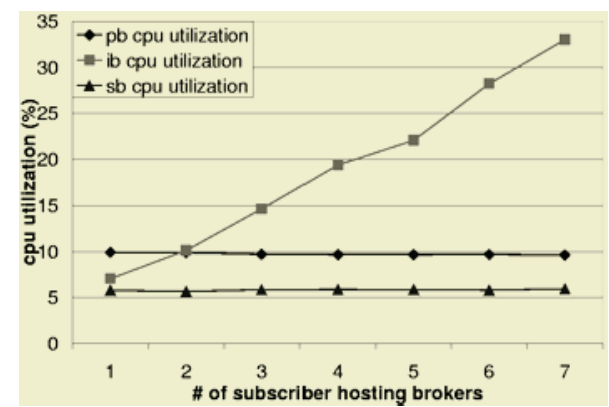

(a) flooding algorithm

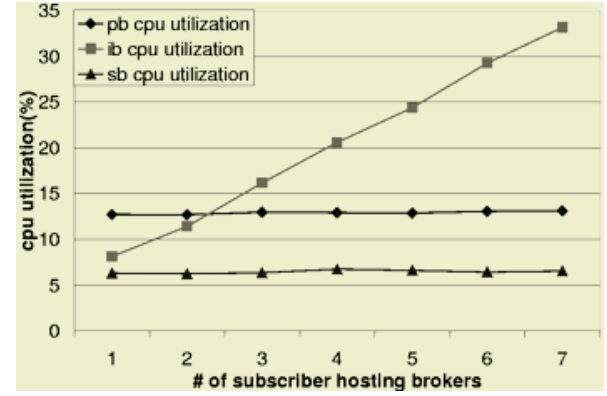

(b) subscription propagation

Fig. 6. Scalability: Broker CPU Utilization Versus Number of Brokers.

$p b$ to a number of brokers $s b_{1-n}$ for subscribers. Each of these brokers is in a separate routing tree node. We vary the number $n$ of $s b$ 's.

Messages are published through $p b$ at a fixed rate of 2000 messages/second throughout the test. We demonstrate the result in a setup that is not favorable to subscription propagation to show the low overhead incurred by it compared with the flooding scheme. We set up each published message to be subscribed by some subscriber at each $s b$. Subscribers are evenly distributed onto $s b$ 's. Each $s b$ has two groups of subscribers with each group receiving 10000 messages/second. One group is steady and the other is dynamic with periodic unsubscriptions followed immediately by re-subscriptions. Every 2 seconds on average, an unsubscription/subscription occurs at a $s b$ and causes the $s b$ virtual time to advance by 2 . Thus, this simulates the situation where the broker virtual time advances by one every second at each $s b$. In situations where subscription/unsubscription occurs more frequently, they could be batched to reduce the rate at which the $s b$ virtual times advance. Figure 6 shows the CPU utilization at $p b, i b$ and $s b_{1-n}$ (averaged).

In both the flooding scheme and with subscription propagation (Figure 6(a) $\&(\mathrm{~b}))$, CPU utilization at $p b$ and $s b_{1-n}$ stays constant with $n$ changing from $1-7$. This is due to the Vector-Digest and caching scheme we described. The 


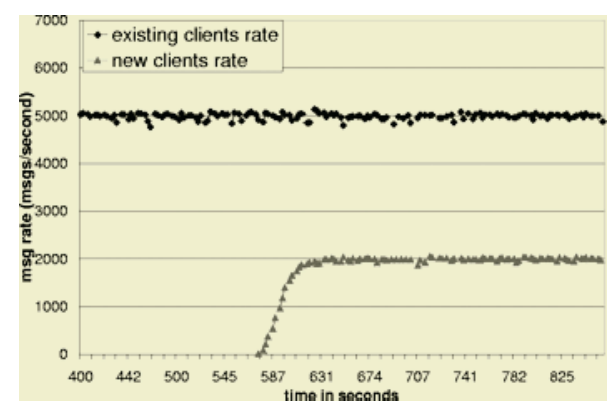

(a) subscriber message rate

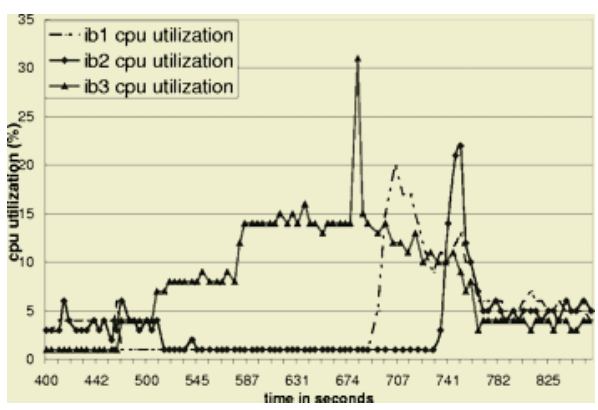

(b) CPU utilization at $i b_{1-3}$

Fig. 7. Client message rate and intermediate broker CPU utilization with crash failure.

CPU differences at $i b$ and $s b$ 's between the two schemes are also very small. There are $(3 \%)$ differences on the $p b$ CPU utilization. This is due to the sophisticated message encoding used in Gryphon. As $p b$ assigns $V_{m}$ digest to a message, the message has to be re-encoded and this is not needed with flooding in Gryphon. Such difference can be eliminated by encoding optimizations.

Failure Test. This test demonstrates the lightweight failover characteristics of our approach. Even in the absence of a majority of brokers in a cell, our algorithm is able to accept new subscriptions and deliver messages for them. When a path fails, the system switches to the remaining available paths and provides continuing service.

The test is set up as a redundant routing tree of 4 nodes and 6 brokers: broker $p b$ for publishers and $s b_{1-2}$ for subscribers, each in a separate node $N_{p b}$, $N_{s b_{1}}$ and $N_{s b_{2}}$. Broker $i b_{1-3}$ are all in one node $N_{i b}$. Node $N_{p b}$ is connected to $N_{i b}$, which further connects to $N_{s b_{1}}$ and $N_{s b_{2}}$.

In this test, traffic from $p b$ to $s b_{1-2}$ is shared among $i b_{1-3}$. Messages are published through $p b$ at a rate of 2000 messages per second on 100 topics. Initially, there are 2 groups of clients connected to $s b_{1}$ and 1 group to $s b_{2}$, each group with 250 subscribers evenly distributed among the first 50 topics. Thus, the aggregated message rate per group is 5000. A fourth group of 100 clients to the remaining 50 topics connect at a later time. The aggregated message rate for this group is 2000. Figure 7 (a) shows the message rates for one of the first 3 groups and the fourth group. Figure $7(\mathrm{~b})$ shows the CPU utilization at $i b_{1-3}$.

At time 400, since only the first 50 topics are subscribed to, the messages are routed through $i b_{1}$ and $i b_{2}$. Broker $i b_{3}$ is not used because of the simple hashing scheme used for load balancing. At time $475, i b_{1}$ crashes, the system fails over to $i b_{3}$, and CPU utilization at $i b_{3}$ increases to $4 \%$ to the same as $i b_{2}$. About 30 seconds later, at time 505, $i b_{2}$ crashes, and all messages on the first 50 topics are routed through $i b_{3}$. CPU utilization at $i b_{3}$ doubles to $8 \%$. During these routing changes the client message rate is not affected. At time 565, a new group of 100 subscribers to the latter 50 topics starts to connect. Even though only $i b_{3}$ is 
available, our approach is able to make progress and starts to deliver messages for the new clients. When $i b_{1}$ and $i b_{2}$ recover about 130 and 160 seconds later at time 691 and 731, traffic is once again shared among the available paths. During this process, service to clients is not affected as their message rate stays constant.

\section{Related Work}

In this section we survey previous work on subscription propagation and aggregation in publish/subscribe systems. Techniques for subscription aggregation ([12]) are complimentary to our work.

Siena [5] and XNet [6] support subscription propagation and aggregation to achieve scalability. Their topology has redundancy, with multiple routes between servers. However, the subscriptions are only propagated along a single selected "best route" in a spanning tree. If a failure occurs on the selected path, the system must select another path and subscription information need to be set up for the new path before message routing can be resumed. As a result, recovery from a spanning tree link failure is slow. In addition, these works do not address the support of reliable delivery.

Elvin [17] is mainly designed around a single server that filters and forwards producer messages directly to consumers. It doesn't have a scalable solution for multiple servers.

Snoeren et. al [18] propose an approach for improving reliability and low latency by sending simultaneously over redundant links in a mesh-based overlay networks. The protocol does content-based routing and provides high level of availability. However, there is no guarantee of reliable delivery when subscriptions are dynamically added and removed.

REBECA $[12,13]$ supports subscription propagation and aggregation over a network constructed as a tree of brokers. Their subscription aggregation techniques, such as filter merging, are applicable to our work. The system has a self-stabilization component that uses time based leases to validate routing entries in brokers. This is a viable technique for best-effort delivery, but does not support reliable delivery since it is possible for a broker to filter a message that is relevant for a downstream subscriber.

JEDI [7] guarantees causal ordering of events. Their distributed version of event dispatcher constitutes of a set of dispatching servers interconnected into a tree structure. This distributed version, while addressing part of the need of Internet-wide distributed applications engaging in an intense communication, does not accommodate and utilize redundant links between dispatching servers and hence is not highly available and easy for load sharing.

Tapestry [20] provides fault tolerant routing by dynamically switching traffic onto precomputed alternate routes. Messages in Tapestry can be duplicated and multicast "around" network congestion and failure hotspots with rapid reconvergence to drop duplicates. However, it does not support content routing. 
Scribe [15] is a large-scale and fully decentralized event notification system built on top of Pastry - a peer-to-peer object location and routing substrate overlayed on the Internet. It leverages the scalability, locality, fault-resilience and self-organization properties of Pastry. However, Scribe does not support content-based routing and wild card topic subscriptions . The system builds separate multicast trees for individual topics using a scheme similar to reverse path forwarding and inverts the subscription message path for later event distribution. This makes it impossible to add a node to the multicast tree for load sharing. The system recovers from multicast node failures by building new trees. It does not support reliable delivery, and unsubscription has to be delayed until the first event is received.

Triantafillou et. al [19] proposed an approach to propagating subscription summaries and performing event matching and routing. Their subscription propagation algorithm, which affects the way events are routed, requires each broker to have global knowledge on the broker network topology. In addition, the approach does not support reliable delivery.

Since Lamport's work on logical time and clocks [11], significant work has been done using logical time such as virtual time [9], version vectors [14], vector times [16] and multipart timestamps [10]. Our work shares the property that logical time vectors are used as a concise form for representing large information. However, these works are focused on detecting state inconsistencies or causal relationships, which is only part of the problem subscription propagation is facing in $\mathrm{pub} / \mathrm{sub}$ systems.

\section{Conclusions}

We have developed algorithms supporting subscription propagation with high availability, easy load-sharing and light failover in a content-based pub/sub system over a redundant overlay network. The algorithm does not require agreements or quorum among redundant brokers. We also presented the experiment results that show the high performance, scalability, low latency and availability. Future work includes investigation into support for event advertisement ([5]), adaptively applying subscription propagation according to different subscription profile and locality.

\section{References}

1. Java (tm) message service. In http://java.sun.com/products/jms/.

2. M. K. Aguilera, R. E. Strom, D. C. Sturman, M. Astley, and T. D. Chandra. Matching events in a content-based subscription system. In Proceedings of the Principles of Distributed Computing, 1999, pages 53-61, May 1999.

3. S. Bhola, R. Strom, S. Bagchi, Y. Zhao, and J. Auerbach. Exactly-once delivery in a content-based publish-subscribe system. In Proceedings of the International Conference on Dependable Systems and Networks (DSN'2002), pages 7-16, 2002. 
4. S. Bhola, Y. Zhao, and J. Auerbach. Scalably supporting durable subscriptions in a publish/subscribe system. In Proceedings of the International Conference on Dependable Systems and Networks (DSN'2003), pages 57-66, 2003.

5. A. Carzaniga, D. Rosenblum, and A. Wolf. Design and evaluation of a wide-area event notification service. ACM Transactions on Computer Systems, 19(3):332383, August 2001.

6. R. Chand and P. Felber. A scalable protocol for content-based routing in overlay networks. In Proceedings of the IEEE International Symposium on Network Computing and Applications (NCA'03), Cambridge, MA, April 2003.

7. G. Cugola, E. D. Nitto, and A. Fuggetta. The jedi event-based infrastructure and its application to the development of the opss wfms. IEEE Transactions on Software Engineering, 27(9):827-850, September 2001.

8. F. Fabret and et. al. Filtering algorithms and implementation for very fast publish/ subscribe systems. SIGMOD Record (ACM Special Interest Group on Management of Data), 30(2):115-126, 2001.

9. D. Jefferson. Virtual time. ACM Transactions on Programming Languages and Systems, 7(3):404-425, 1985.

10. R. Ladin, B. Liskov, and L. Shrira. Lazy replication: Exploiting the semantics of distributed services. In ACM Symposium on Principles of Distributed Computing, 1990.

11. L. Lamport. Time, clock, and the ordering of events in a distributed system. Communications of the ACM, 21:558-565, 1978.

12. G. Mühl. Large-Scale Content-Based Publish/Subscribe Systems. PhD thesis, Darmstadt University of Technology, September 2002.

13. G. Mühl, L. Fiege, and A. P. Buchmann. Filter similarities in content-based publish/subscribe systems. In Proceedings of International Conference on Architecture of Computing Systems (ARCS'02), 2002.

14. D. Parker and et.al. Detection of mutual inconsistency in distributed systems. IEEE Transactions on Software Engineering, SE-9(3):240-247, 1983.

15. A. Rowstron, A. Kermarrec, M. Castro, and P. Druschel. Scribe: The design of a large-scale event notification infrastructure. In Proceedings of 3rd International Workshop on Networked Group Communication (NGC 2001), UCL, London, UK, November 2001.

16. R. Schwarz and F. Mattern. Detecting causal relationships in distributed computations: In search of the holy grail. Distributed Computing, pages 149-174, 1994.

17. B. Segall, D. Arnold, J. Boot, M. Henderson, and T. Phelps. Content based routing with elvin4. In Proceedings of AUUG2K, Canberra, Australia, April 2000.

18. A. Snoeren, K. Conley, and D. Gifford. Mesh-based content routing using xml. In Proceedings of the 18th ACM Symposium on Operating System Principles, 2001.

19. P. Triantafillou and A. Economides. Subscription summarization: A new paradigm for efficient publish/subscribe systems. In Proceedings of the 24th International Conference on Distributed Computing Systems (ICDCS'04), 2004.

20. B. Zhao, L. Huang, A. Joseph, and J. Kubiatowicz. Exploiting routing redundancy using a wide-area overlay. Technical Report UCB/CSD-02-1215, University of California, Berkeley, 2002. 